\title{
Pathogenesis and management of tendinopathies in sports medicine
}

\author{
M. P. Mead' | J. P. Gumucio ${ }^{1,2}$ | T. M. Awan' | C. L. Mendias ${ }^{1,2}$ (iD | K. B. Sugg ${ }^{1,2,3}$ (iD)
}

${ }^{1}$ Departments of Orthopaedic Surgery, Section of Plastic \& Reconstructive Surgery, University of Michigan Medical School, Ann Arbor, MI, USA

${ }^{2}$ Departments of Molecular \& Integrative Physiology, Section of Plastic \& Reconstructive Surgery, University of Michigan Medical School, Ann Arbor, MI, USA

${ }^{3}$ Departments of Surgery, Section of Plastic \& Reconstructive Surgery, University of Michigan Medical School, Ann Arbor, MI, USA

\section{Correspondence}

Kristoffer B. Sugg, Department of Surgery, Section of Plastic \&

Reconstructive Surgery, University of Michigan Medical School, Ann Arbor, MI, USA.

Email: krisugg@med.umich.edu

\section{Funding information}

National Institute of Arthritis and

Musculoskeletal and Skin Diseases, Grant/

Award Number: F31-AR065931, F32-

AR067086, R01-AR063649
Tendinopathy is a major clinical problem in sports medicine and is often difficult to treat. Traditional therapeutic approaches have focused on reducing inflammation, yet research suggests that little to no inflammation is present in the tendons that fail to heal. The purpose of this review was to evaluate the effectiveness of the available treatment options for tendinopathy and to inform best clinical practices. A comprehensive search of electronic databases (PubMed, Google Scholar, and Web of Science) was conducted to identify relevant studies through June 2016. Studies were deemed relevant if they were published in English and contained original research on the management of tendinopathy in humans. Studies varied in methodological quality and were often limited by small sample size and lack of sufficient control groups. Critical evaluation of the literature suggests that physical therapy with or without eccentric exercise should be considered a first-line treatment. Corticosteroids and non-steroidal anti-inflammatory drugs provide short-term symptomatic relief, but long-term efficacy has not been demonstrated. Inconsistent results do not support the routine use of prolotherapy, platelet-rich plasma injections, and topical nitric oxide patches. Operative intervention should be reserved until conservative measures fail or an obvious operative lesion is present. While numerous therapeutic modalities exist for tendinopathy in the athlete, the ideal treatment protocol has not been clearly defined. The development of new targeted therapies for tendinopathy is likely to follow a greater understanding of the cellular and molecular mechanisms that underlie its pathogenesis.

\section{K E Y W O R D S}

tendinitis, tendinopathy, tendinosis, tendon injury, wound healing

\section{1 | INTRODUCTION}

Tendinopathy is a frequent and difficult-to-treat clinical problem in athletes and invariably leads to a negative impact on sport performance. It is often considered a failure of damaged matrix proteins within the tendon to properly heal, but the exact etiology remains largely unknown. Hallmark findings of this disorder include localized pain, swelling, and reduced strength and performance of the affected tendon. While frequently thought to be associated with increasing age and participation in sports, tendinopathy is routinely diagnosed in patients of all ages and levels of physical activity. ${ }^{1}$ Management of this painful condition can be particularly challenging, and recovery is usually slow. ${ }^{2}$ This is especially true for athletes where higher levels of physical activity and minimal rest times make it difficult to return to sports and can lead to re-injury. Numerous treatment options exist for tendinopathy, but overall, there is a lack of consensus in the literature regarding their efficacy. Indeed, some tendinopathies respond to 
simple interventions, while others are refractory to nearly all forms of treatment. A greater understanding of the cellular and molecular mechanisms that underlie the pathogenesis of tendinopathy will likely facilitate the discovery of new targeted therapies to enhance tendon healing and repair.

The objective of this review was to first explore the normal structure and function of tendon followed by an overview of tendinopathy, including its classification, epidemiology, and the risk factors associated with its development. The remainder of this review will then provide a cogent and practical approach to the management of tendinopathy with a focus on both non-operative and operative therapeutic interventions.

\section{2 | TENDON STRUCTURE AND CELL BIOLOGY}

\section{1 | Macroscopic overview of tendon}

Tendons connect skeletal muscle to bone and transmit force generated by muscular contraction to permit movement. They are considered an extension of the skeletal muscle extracellular matrix (ECM) and terminate on bone at the enthesis. ${ }^{3}$ Tendons vary in size and shape with short, thick tendons originating from the powerful muscle groups of the proximal limb, while long, thin tendons execute fine movements of the digits. ${ }^{4}$ Smooth and efficient motion requires tendon gliding that is not restricted by adjacent tissue. For that reason, synovial sheaths form a closed system around many tendons to provide lubrication and to cushion the tendon as it stretches and relaxes. ${ }^{3}$ Tendons that lack true synovial sheaths are instead surrounded by a loose, fatty, and vascularized peritendinous tissue, which allows for free excursion of the tendon within its fascial compartment. ${ }^{4}$

The mechanical properties of tendon are determined by the macromolecular structural organization and biochemical composition of the ECM. ${ }^{5}$ When a tendon is loaded, the extent of tissue deformation is dependent on the rate at which the load is applied. ${ }^{6}$ At low strain rates, tendons deform more and absorb more energy, while at high strain rates, tendons deform less, become stiffer, and are more effective at carrying mechanical load. ${ }^{7}$ During physical activity, tendons store elastic energy as they are stretched and release that energy as they shorten. As tendons are stretched, the non-covalent bonds between amino acid residues are broken which gives off energy in the form of heat. ${ }^{6}$ In situations of high-frequency cyclical stretching, certain regions within tendon may not be able to efficiently dissipate this heat. ${ }^{6}$ This potentially causes an irreversible denaturing of matrix proteins and can lead to tendinopathy or acute tendon rupture. ${ }^{8}$ In addition to the rate at which tendons are stretched, the degree to which tendons are stretched can also contribute to injury. Strains $<4 \%$ generally allow the tendon to return to its original length once the load is removed, but above $4 \%$ strain the collagen fibers can begin to fail, and beyond $8 \%$ strain the tendon is susceptible to rupture. ${ }^{2,4}$ Lastly, 2 additional features that highlight the viscoelastic properties of tendon are creep and stress relaxation. Creep refers to time-dependent elongation of the tendon while under constant load, whereas stress relaxation is a time-dependent decline in the load required to maintain constant elongation of the tendon. ${ }^{9}$

\subsection{Microscopic overview of tendon}

Tendon is a hypocellular tissue composed of elongated fibroblasts interspersed between a complex network of matrix proteins. The organizational pattern of tendon closely resembles that of skeletal muscle with distinct connective tissue layers running parallel to its longitudinal axis. ${ }^{4}$ The smallest functional unit of tendon is the collagen fibril. Collagen fibrils combine to form larger collagen fibers, and then, groups of these fibers coalesce into tendon fascicles. Tendon fascicles are enveloped by the endotenon, which is a delicate reticular network of connective tissue supporting a rich supply of vascular, lymphatic, and neural channels. ${ }^{4}$ The tendon proper is the final structure formed by numerous tendon fascicles held together by a loose epithelial-like tissue layer called the epitenon. ${ }^{10}$ The epitenon contains larger blood vessels and nerves and is a potential source of new tendon fibroblasts during periods of tendon growth and repair. ${ }^{11}$

During the course of embryonic development, tendons arise from a separate pool of progenitor cells than other limb tissues, including skeletal muscle, cartilage, and bone. ${ }^{12}$ Tendon fibroblasts are rod- or spindle-shaped cells with long cytoplasmic extensions known as fibripositors, whose primary function is to synthesize and secrete collagen fibrils in parallel with the direction of load across the long axis of the tendon. ${ }^{13}$ Ippolito and colleagues originally described 2 different populations of tendon fibroblasts, namely immature proliferating tenoblasts and terminally differentiated tenocytes. ${ }^{14}$ As individuals approach adulthood, the amount of tenoblasts decrease while terminally differentiated tenocytes become the most abundant cell population in tendon. ${ }^{15}$ The origin and identity of the stem cell population within tendon that gives rise to tenocytes is not fully understood. However, pericytes, which are a population of stem cells that exist in close proximity to the vasculature, appear to be attractive candidates. ${ }^{16}$ The remaining cells within tendon mostly consist of endothelial and smooth muscle cells of the tendon vasculature, synovial cells within the tendon sheath, and chondrocytes at the enthesis. ${ }^{2}$ 
The main structural protein of tendon is type I collagen, which accounts for $65 \%-80 \%$ of the tendon dry weight. ${ }^{5}$ Type I collagen is first synthesized as a procollagen molecule within the tendon fibroblast and typically consists of 2 $\alpha 1$ and $1 \alpha 2$ subunits. Procollagen is then secreted from the cell into the tendon ECM where its amino- and carboxy-terminal ends are cleaved to produce tropocollagen. As the final step in the collagen synthesis pathway, multiple tropocollagen molecules are cross-linked resulting in the formation of the mature collagen fibril. Type I and III collagens are part of the fibrillar collagen family and play an important role in the longitudinal transmission of force during locomotion. ${ }^{3}$ Compared to type I collagen, type III collagen tends to be smaller in diameter, less organized, and has decreased tensile strength. ${ }^{17}$ Type III collagen is often found at increased levels in aged tendon or at the rupture sites of highly stressed tendons. ${ }^{18}$ Type $\mathrm{V}$ collagen is also a fibrillar collagen, but is present in lesser quantities and regulates the initiation of collagen fibril assembly. ${ }^{19}$ In addition to the fibrillar collagens, many other types of collagen can be found in tendon. These collagens, such as the network type IV and VI collagens, help to transmit forces laterally between cells and provide structural support for vascular and lymphatic tissue in the endotenon and epitenon. ${ }^{3}$ Mice deficient in type VI collagen display mechanically weak tendons with an increase in the number of small and aberrant collagen fibrils, indicating that type VI collagen also facilitates collagen fibril organization. ${ }^{20}$ Aside from collagen, a small but significant portion of the tendon structure in humans is formed by elastin. ${ }^{4}$ While little is known about its exact function, elastin tends to be localized to the interfascicular matrix and likely contributes to sliding between adjacent tendon fascicles and recoil after mechanical loading. ${ }^{21}$ Finally, intermixed with the collagen and non-collagen proteins of the tendon ECM is the gelatinous ground substance composed of proteoglycans (PGs) and glycosaminoglycans (GAGs). PGs and GAGs have a fixed net negative charge that allows them to retain water, which in turn helps the tendon to dissipate heat into the surrounding tissues as well as resist high compressive and tensile forces during exercise. ${ }^{22}$

In humans, the core of the Achilles tendon will grow until the age of 17, after which protein synthesis and matrix turnover begin to decline. ${ }^{23}$ This is supported by animal studies in rodents that demonstrate tendon growth mostly occurs in an outward direction from the epitenon. ${ }^{15,16}$ However, the lack of a discernible endotenon in rodents makes it difficult to rule out the possibility of intrafascicular growth between tendon fascicles. Together, these findings suggest that the lack of tissue renewal in the core of adult tendon tissue may contribute to the protracted course that is characteristic of many tendinopathies, and perhaps cell-based therapies that selectively utilize a fraction of cells from those connective tissue layers known to be enriched in tendon stem cells may enhance the regeneration of injured tendon tissue.

\section{3 | EPIDEMIOLOGY AND PATHOGENESIS OF TENDINOPATHY}

Tendon injuries account for $30 \%-50 \%$ of all injuries in sports, and the risk of these injuries is increasing as a larger proportion of younger and older age groups participate in recreational activities. ${ }^{1}$ Greater demands have been placed on athletic performance, requiring that athletes train longer, harder, and more often. This type of chronic overuse is associated with an increased risk of developing tendinopathy. For example, Olympic runners had an increased incidence of Achilles tendinopathy with an adjusted odds ratio of 31.2 compared with age-matched controls. ${ }^{24}$ In addition to the frequency of overuse, activities that prolong the loading of tendons without adequate rest in between sessions also contribute to the risk of developing tendinopathy. ${ }^{25}$ It has been demonstrated that total hours spent practicing sports is a known risk factor for developing patellar tendinopathy in elite soccer players. ${ }^{26}$

Understanding the pathogenesis of tendinopathy has been hindered by a lack of consistent terminology. Traditionally, the term tendinitis referred to symptomatic tendons associated with chronic pain and implied an element of inflammation in its basic etiology, especially in the initial phases of the disease process. ${ }^{27}$ Repetitive microtrauma from overload or overuse can lead to collagen fibril rupture and activation of the innate immune system. ${ }^{27}$ However, continued research has demonstrated little to no biochemical or histological evidence of inflammation in biopsies from patients with chronic tendinopathy. ${ }^{28}$ Given the uncertainty surrounding the role of inflammation in the development of tendon disorders, we prefer to use the term tendinopathy to describe an ongoing process of degeneration and failed regeneration of the tendon ECM in response to injury or disease. In clinical practice, tendinopathy refers to a spectrum of pathologies from intratendinous degenerative lesions that are a source of chronic pain, to spontaneous tendon rupture as a result of mechanical attrition.

Several features are characteristic of tendinopathy at the cellular level (Figure 1). Compared to healthy tendons, tendinopathic tendons have marked disorganization and separation of collagen fibrils with a concomitant increase in mucoid ground substance. ${ }^{29}$ Collagen fibril diameter is more variable, and an increased content of type III collagen contributes to mechanical weakness of the diseased tendon. ${ }^{18}$ Tendon fibroblasts adopt a rounded rather than flattened appearance and tend to be unevenly distributed throughout the tissue. Another hallmark feature of 

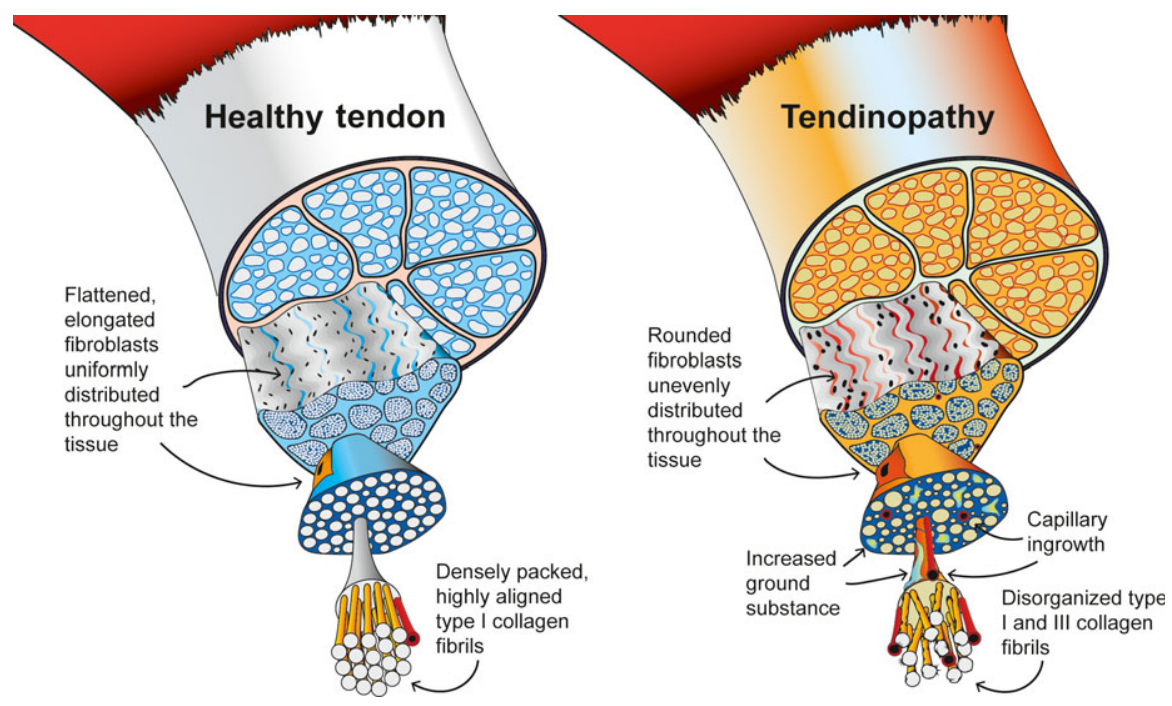

FIGURE 1 Graphical representation of the morphological features in healthy tendon and tendinopathy. Modified from Scott et $\mathrm{al}^{64}$

tendinopathy is neovascularization with budding capillaries invading the tendon from the paratenon. ${ }^{29}$ These capillaries are usually accompanied by the ingrowth of sensory nerve fibers that release nociceptive substances and trigger pain. ${ }^{30}$ Finally, there is absence of an inflammatory cell infiltrate in the tendon proper, although inflammation can be observed within the peritendinous tissue. ${ }^{29}$

\section{4 | NON-OPERATIVE MANAGEMENT OF TENDINOPATHY}

The management of tendinopathy can vary based on the history and extent of the condition, the severity of symptoms, and the physical demands and activity level of an athlete. Conservative measures remain the mainstay of treatment for most tendinopathies, but operative intervention is available for recalcitrant cases, or for athletes with obvious operative lesions (Table 1).

\section{1 | Eccentric exercises}

Eccentric exercises, defined as exercises in which the muscle and tendon lengthen while under tension, have been well studied and long utilized as a form of treatment in the management of tendinopathies. ${ }^{31}$ Several studies have demonstrated that eccentric exercises can improve patientreported outcomes with favorable changes in strength, clinical imaging, and biochemical and histological markers of ECM remodeling. ${ }^{32,33}$ While the mechanisms are not completely understood at the molecular level, eccentric exercises result in the production of new type I collagen and increase the density of collagen fibrils. ${ }^{34}$ Furthermore, mechanical loading induces the expression of genes such as scleraxis and tenomodulin that are associated with the accumulation of new tenocytes and the production of healthy ECM. ${ }^{15,16} \mathrm{~A}$ particular form of eccentric exercise, termed "heavy slow resistance" training, uses heavy weight with slow repetition and has been notably effective for the treatment of patellar tendinopathy. ${ }^{33}$ The combined efficacy of eccentric training from clinical research studies, the low cost of therapy, and the compelling evidence of improved ECM quality at the cellular and molecular levels have resulted in eccentric exercises being frequently considered as a first-line therapy in the management of tendinopathy.

\section{2 | Non-steroidal anti-inflammatory drugs (NSAIDs)}

Non-steroidal anti-inflammatory drugs, which function to block the production of prostaglandins from the cyclooxygenase enzymes, remain a popular choice for the management of tendinopathies. Prostaglandins are physiologically active lipid molecules derived from arachidonic acid that play an important role in the development of acute inflammation. Accordingly, both the oral and topical delivery of NSAIDs are generally effective as short-term analgesics for these painful conditions. ${ }^{35,36}$ While useful for reducing pain and inflammation, NSAIDs do not appear to alter the course of the disease process, which is consistent with the lack of an inflammatory phenotype in tendinopathic tendons. $^{29,37}$ Furthermore, the drugs likely do not accumulate in sufficient quantities within the tendon tissue to change gene transcription. ${ }^{38}$ There is also some evidence to suggest that NSAIDs may negatively impact tendon healing by impairing the proliferation and differentiation of tenocytes, as well as promoting the formation of adipocytes from mesenchymal stem cells. ${ }^{39,40}$ In summary, NSAIDs may have utility in the alleviation of acute pain, but emerging evidence indicates no effect, or potential deleterious effects of NSAIDs, in the management of tendinopathy. 
TA B LE 1 Summary of non-operative and operative therapeutic interventions for the management of tendinopathy

\begin{tabular}{|c|c|c|}
\hline Treatment & Proposed mechanism & Outcome \\
\hline \multicolumn{3}{|l|}{ Non-operative } \\
\hline Eccentric exercises & $\begin{array}{l}\text { Promotion of tissue reorganization with } \\
\text { improved changes in tendon structure and } \\
\text { mechanical properties }{ }^{31,34}\end{array}$ & $\begin{array}{l}\text { Positive outcomes including reduced pain, } \\
\text { improved strength, and decreased time to return to sport } \\
32,33\end{array}$ \\
\hline NSAIDs & $\begin{array}{l}\text { Reduced pain and inflammation } \\
\text { during tendon healing } \\
37\end{array}$ & $\begin{array}{l}\text { Positive short-term outcomes for analgesia }{ }^{35,36} \text {; no } \\
\text { significant long-term benefits }{ }^{29,37} \text {; some evidence to } \\
\text { support that NSAIDs have potential to inhibit normal } \\
\text { tendon healing }{ }^{39,40}\end{array}$ \\
\hline Corticosteroids & $\begin{array}{l}\text { Reduced pain and inflammation } \\
\text { within the peritendinous tissue }\end{array}$ & $\begin{array}{l}\text { Positive short-term outcomes for analgesia and } \\
\text { functional improvement }{ }^{33,41-43} \text {; limited or no long-term } \\
\text { benefits }^{45} \text {; potential risk of complications }{ }^{44}\end{array}$ \\
\hline Nitric oxide & $\begin{array}{l}\text { Increased tenocyte proliferation } \\
\text { and collagen synthesis }{ }^{47}\end{array}$ & $\begin{array}{l}\text { Unclear short-term outcomes }{ }^{50,51} \text {; no significant long-term } \\
\text { benefits }^{52}\end{array}$ \\
\hline PRP & $\begin{array}{l}\text { Increased growth factors and cytokines } \\
\text { at the side of tendon healing }\end{array}$ & $\begin{array}{l}\text { Insufficient studies to demonstrate short- or } \\
\text { long-term benefits } 57,58\end{array}$ \\
\hline \multicolumn{3}{|l|}{ Operative } \\
\hline $\begin{array}{l}\text { Percutaneous } \\
\text { longitudinal tenotomy }\end{array}$ & $\begin{array}{l}\text { Promotion of tissue regeneration } \\
\text { and angiogenesis }\end{array}$ & Positive long-term outcomes with minimal complications ${ }^{60}$ \\
\hline $\begin{array}{l}\text { Percutaneous } \\
\text { ultrasonic } \\
\text { microtenotomy }\end{array}$ & $\begin{array}{l}\text { Removal of pathological tissue and } \\
\text { sensory nerve fiber destruction }\end{array}$ & Reduced pain symptoms at $3 y^{63}$ \\
\hline
\end{tabular}

NSAIDs, non-steroidal anti-inflammatory drugs; PRP, platelet-rich plasma.

\section{3 $\mid$ Corticosteroids}

Corticosteroids are synthetic analogues of a particular class of steroid hormones produced by the adrenal cortex. These potent anti-inflammatory molecules have been evaluated extensively in many clinical trials of patients with tendinopathies. ${ }^{41,42}$ Even though the inflammatory process appears to play little to no role in the pathogenesis of tendinopathy, several studies have demonstrated that corticosteroid therapy often significantly improves patient outcomes in the short term by providing pain relief and enhancing function. ${ }^{43}$ The positive effect of corticosteroids on pain and function is thought to occur due to a reduction in inflammation within the peritendinous tissue. ${ }^{33}$ However, there exists the potential risk of iatrogenic tendon rupture and atrophy of the surrounding soft tissues with repeated corticosteroid use. ${ }^{44}$ A 2015 Cochrane Review investigating the efficacy of injection therapies including corticosteroids for the treatment of painful Achilles tendons also found that there is insufficient evidence to support the routine use of injection therapies in patients with Achilles tendinopathy. ${ }^{45}$ While corticosteroids have been shown to reduce pain in many tendon disorders, clinicians should be careful against the widespread use of corticosteroids in the management of tendinopathy, due to the lack of longterm benefits and the potential risk of adverse effects.

\section{4 | Nitric oxide}

Nitric oxide therapies have gained popularity within recent years for the management of tendinopathies. ${ }^{46}$ The most common approach involves the transdermal delivery of nitroglycerin to the involved area where it is converted into bioactive nitric oxide by endogenous nitric oxide synthase. ${ }^{46}$ At the cellular and molecular levels, nitric oxide increases tenocyte proliferation and collagen synthesis, while the inhibition of nitric oxide synthase decreases the cross-sectional area of healing tendons and reduces the amount of mechanical load required for tendon failure. ${ }^{47}$ There have been several randomized controlled trials to evaluate the effectiveness of topical nitroglycerin in patients with tendinopathy, but have yielded conflicting results. ${ }^{4,49}$ Two meta-analyses have concluded that while additional studies need to be performed, there is evidence to support the use of low-dose nitroglycerin patches for the short-term relief of pain associated with tendinopathy. ${ }^{50,51}$ Studies of the long-term efficacy of nitroglycerin patches, however, have generally shown marginal to no improvement in pain and function. ${ }^{52}$ Side effects of nitroglycerin patches have also been reported, including headache, rash, facial flushing, and sweating. ${ }^{49}$ These potential side effects along with the contradictory short-term results and the lack of long-term efficacy have limited the 
enthusiasm for the use of nitroglycerin patches as a first-line therapy in the management of tendinopathy.

\subsection{Prolotherapy}

Prolotherapy refers to the injection of an irritant solution within or around a tendinopathic tendon..$^{53}$ The irritant effect is thought to promote inflammation and trigger a regenerative response in order to heal the chronically degraded ECM that is characteristic of patients with tendinopathy. There is considerable variability in the type and concentration of irritant solution that can be injected, although most solutions contain either hyperosmolar dextrose, phenol-glycerin-glucose, or sodium morrhuate. While the mechanism of action is not completely understood, hyperosmolar dextrose is thought to dehydrate cells until they rupture by creating a large osmotic gradient, phenol-glycerin-glucose causes localized cellular and tissue irritation, and sodium morrhuate functions as a chemoattractant for inflammatory cells and other mediators. ${ }^{53}$ These irritant solutions can also sclerose small blood vessels and nociceptive neurons that are enriched in tendinopathic tendons. A small number of clinical studies have evaluated the effectiveness of prolotherapy in patients with tendinopathy, but most of these studies are limited by either small sample size or lack of appropriate controls. ${ }^{54,55}$ Therefore, while prolotherapy is a relatively safe and low-cost treatment option, there is a need for larger randomized controlled trials to more rigorously assess the use of prolotherapy as a first-line therapy in the management of tendinopathy.

\section{6 | Platelet-rich plasma (PRP)}

Platelet-rich plasma is an autologous blood product consisting of plasma enriched with a concentration of platelets that is greater than that of whole blood, typically at least 4 times the baseline value. ${ }^{56}$ PRP has grown in popularity within recent years as a way to accelerate healing in a variety of musculo-skeletal conditions, and numerous potential applications have been explored, including the use of PRP as a treatment option for tendinopathy. Platelets are thought to be particularly important for injured tissues to properly heal, as they are enriched with multiple growth factors and cytokines, including transforming growth factor beta (TGF$\beta$ ), interleukins (ILs), fibroblast growth factors (FGFs), platelet-derived growth factors (PDGFs), vascular endothelial growth factors (VEGFs), connective tissue growth factor (CTGF), and others. ${ }^{56}$ These proteins are thought to enhance tissue healing when delivered locally. ${ }^{2}$ However, recent work using wide-scale genome profiling and bioinformatics has reported PRP to work through inducing local inflammation in tissue. ${ }^{56}$

There have been several clinical studies to explore the effectiveness of PRP in treating patients with tendinopathy, but have produced mixed results. ${ }^{57,58}$ Some of the most encouraging results have come in the treatment of lateral epicondylitis, with PRP demonstrating a near twofold improvement in outcomes compared to corticosteroids. ${ }^{57} \mathrm{~A}$ recent meta-analysis concluded that PRP injections did not provide significantly greater relief compared to placebo or dry needling for the treatment of tendinopathy at 6 months of follow-up, although there was marginal improvement in patients suffering from rotator cuff tendinopathy. ${ }^{59}$ Larger clinical studies, conducted on multiple different anatomical locations, are necessary to better inform the evidence-based use of PRP in the management of tendinopathy.

\section{5 | OPERATIVE MANAGEMENT OF TENDINOPATHY}

Nearly a third of all patients with tendinopathy fail nonoperative management. ${ }^{1}$ For these patients, they either alter or reduce their activity level and sport participation, or seek operative treatments. The goal of operative management for patients with tendinopathy is to either remove the pathological areas of tendon or to induce low-grade trauma to the degenerated tendon in an effort to restart the healing response, similar to prolotherapy or PRP. Several techniques are outlined below.

\subsection{Percutaneous longitudinal tenotomy}

Percutaneous longitudinal tenotomy is a procedure in which the surgeon introduces 1 or multiple stab incisions parallel to the longitudinal axis of the tendon. This has been shown to promote tissue regeneration, increase blood flow, and create a local environment more suitable for healing. ${ }^{60}$ This is a relatively simple procedure, which can be performed on an outpatient basis with the use of local anesthesia. ${ }^{61}$ Ultrasound is used to identify the pathogenic area and helps with placement of the stab incisions. Complications from percutaneous longitudinal tenotomies are reported to be minimal and do not lead to long-term morbidity. ${ }^{61}$

\section{2 | Percutaneous ultrasonic microtenotomy}

Percutaneous ultrasonic microtenotomy has recently become available as a method to treat tendinopathy. Using ultrasonic energy, this minimally invasive procedure is able to debride pathologic tissue through a needlelike device placed within the tendon. In a case series of 20 patients with recalcitrant lateral epicondylitis, percutaneous ultrasonic microtenotomy demonstrated improvement in pain and function in $95 \%$ of patients treated at 1 year. ${ }^{62} \mathrm{~A}$ follow-up on the same patient population demonstrated sustained clinical efficacy at 3 years, with all patients 
demonstrating a reduction in tendon thickness and 95\% exhibiting a resolution of tendon hypervascularity. ${ }^{63}$ While studies evaluating the effectiveness of percutaneous ultrasonic microtenotomy have largely been limited to lateral epicondylitis, this treatment has the potential to be used for other refractory cases of tendinopathy.

\section{6 | CONCLUSIONS}

Despite their prevalence, tendon disorders remain difficult to treat. While numerous therapeutic modalities exist for tendinopathy in the athlete, the ideal treatment protocol has not been clearly defined. New insights into the pathophysiology of these diseases are constantly evolving, and more sophisticated treatment strategies continue to emerge. The development of new targeted therapies for tendinopathy is likely to follow a greater understanding of the cellular and molecular mechanisms that underlie its pathogenesis. At the moment, non-operative management is the cornerstone of therapy. Minimally invasive procedures have shown early promising results, especially in patients with recalcitrant tendinopathy, but high-quality randomized controlled trials are needed before these procedures can be recommended for routine use.

\section{7 | PRACTICAL IMPLICATIONS}

- Rehabilitation, with a focus on eccentric training, should be a first-line therapy in the treatment of painful tendon disorders.

- NSAIDs can be used for short-term symptomatic relief. However, prolonged use should be avoided due to the risk of complications and the lack of proven long-term benefit.

- Second-line therapies include prolotherapy, PRP injections, and topical nitric oxide patches. As a group, the data for these are inconclusive, but their risks are low and therefore can be recommended for athletes that fail first-line treatment.

- Corticosteroids should be used with caution in the treatment of painful tendon disorders, as the underlying cause likely does not involve inflammatory changes and their use may increase the risk of spontaneous tendon rupture.

- Surgery should only be pursued when conservative measures have failed or an obvious pathologic lesion is present within the tendon.

\section{ACKNOWLEDGEMENTS}

The authors have no financial interest to declare in relation to the content of this article. This work was supported by
NIH Grants R01-AR063649, F31-AR065931, and F32AR067086.

\section{ORCID}

C. L. Mendias (D) http://orcid.org/0000-0002-2384-0171

K. B. Sugg (D) http://orcid.org/0000-0002-0301-4083

\section{REFERENCES}

1. Ackermann PW, Renstrom P. Tendinopathy in sport. Sports Health. 2012;4:193-201.

2. Sharma P, Maffulli N. Tendon injury and tendinopathy: healing and repair. J Bone Joint Surg Am. 2005;87:187-202.

3. Davis ME, Gumucio JP, Sugg KB, et al. MMP inhibition as a potential method to augment the healing of skeletal muscle and tendon extracellular matrix. J Appl Physiol (1985). 2013;115:884891.

4. Kannus P. Structure of the tendon connective tissue. Scand $J$ Med Sci Sports. 2000;10:312-320.

5. Kjaer M. Role of extracellular matrix in adaptation of tendon and skeletal muscle to mechanical loading. Physiol Rev. 2004;84:649698.

6. Paxton JZ, Baar K. Tendon mechanics: the argument heats up. J Appl Physiol (1985). 2007;103:423-424.

7. Wang JHC. Mechanobiology of tendon. J Biomech. 2006;39: 1563-1582.

8. Patterson-Kane JC, Becker DL, Rich T. The pathogenesis of tendon microdamage in athletes: the horse as a natural model for basic cellular research. J Comp Pathol. 2012;147:227-247.

9. Magnusson SP, Hansen P, Kjaer M. Tendon properties in relation to muscular activity and physical training. Scand J Med Sci Sports. 2003;13:211-223.

10. Taylor SH, Al-Youha S, Van Agtmael T, et al. Tendon is covered by a basement membrane epithelium that is required for cell retention and the prevention of adhesion formation. PLoS One. 2011;6:e16337.

11. Sugg KB, Lubardic J, Gumucio JP, et al. Changes in macrophage phenotype and induction of epithelial-to-mesenchymal transition genes following acute Achilles tenotomy and repair. J Orthop Res. 2014;32:944-951.

12. Schweitzer R, Zelzer E, Volk T. Connecting muscles to tendons: tendons and musculoskeletal development in flies and vertebrates. Development. 2010;137:2807-2817.

13. Canty EG, Lu Y, Meadows RS, et al. Coalignment of plasma membrane channels and protrusions (fibripositors) specifies the parallelism of tendon. J Cell Biol. 2004;165:553-563.

14. Ippolito E, Natali PG, Postacchini F, et al. Morphological, immunochemical, and biochemical study of rabbit achilles tendon at various ages. J Bone Joint Surg Am. 1980;62:583-598.

15. Gumucio JP, Phan AC, Ruehlmann DG, et al. Synergist ablation induces rapid tendon growth through the synthesis of a neotendon matrix. J Appl Physiol (1985). 2014;117:1287-1291.

16. Schwartz AJ, Sarver DC, Sugg KB, et al. p38 MAPK signaling in postnatal tendon growth and remodeling. PLoS One. 2015;10: e0120044.

17. Birk DE, Mayne R. Localization of collagen types I, III and V during tendon development. Changes in collagen types I and III 
are correlated with changes in fibril diameter. Eur J Cell Biol. 1997;72:352-361.

18. Eriksen HA, Pajala A, Leppilahti J, et al. Increased content of type III collagen at the rupture site of human Achilles tendon. J Orthop Res. 2002;20:1352-1357.

19. Wenstrup RJ, Florer JB, Brunskill EW, et al. Type V collagen controls the initiation of collagen fibril assembly. J Biol Chem. 2004;279:53331-53337.

20. Izu Y, Ansorge HL, Zhang G, et al. Dysfunctional tendon collagen fibrillogenesis in collagen VI null mice. Matrix Biol. 2011;30:53-61.

21. Screen HR, Berk DE, Kadler KE, et al. Tendon functional extracellular matrix. J Orthop Res. 2015;33:793-799.

22. Yoon JH, Halper J. Tendon proteoglycans: biochemistry and function. J Musculoskelet Neuronal Interact. 2005;5:22-34.

23. Heinemeier KM, Schjerling P, Heinemeier J, et al. Lack of tissue renewal in human adult Achilles tendon is revealed by nuclear bomb (14)C. FASEB J. 2013;27:2074-2079.

24. Kujala UM, Sarna S, Kaprio J. Cumulative incidence of achilles tendon rupture and tendinopathy in male former elite athletes. Clin J Sport Med. 2005;15:133-135.

25. Cook JL, Purdam CR. Is tendon pathology a continuum? A pathology model to explain the clinical presentation of loadinduced tendinopathy. Br J Sports Med. 2009;43:409-416.

26. Hägglund M, Zwerver J, Ekstrand J. Epidemiology of patellar tendinopathy in elite male soccer players. Am J Sports Med. 2011;39:1906-1911.

27. Millar NL, Hueber AJ, Reilly JH, et al. Inflammation is present in early human tendinopathy. Am J Sports Med. 2010;38:20852091.

28. Alfredson H, Forsgren S, Thorsen $\mathrm{K}$, et al. In vivo microdialysis and immunohistochemical analyses of tendon tissue demonstrated high amounts of free glutamate and glutamate NMDAR1 receptors, but no signs of inflammation, in Jumper's knee. J Orthop Res. 2001;19:881-886.

29. Khan KM. Histopathology of common tendinopathies. Update and implications for clinical management. Sports Med. 1999;27:393408.

30. Schubert T, Weidler C, Lerch K, et al. Achilles tendinosis is associated with sprouting of substance $\mathrm{P}$ positive nerve fibres. Ann Rheum Dis. 2005;64:1083-1086.

31. Lorenz D, Reiman M. The role and implementation of eccentric training in athletic rehabilitation: tendinopathy, hamstring strains, and acl reconstruction. Int J Sports Phys Ther. 2011;6:27-44.

32. Gardin A, Movin T, Svensson L, et al. The long-term clinical and MRI results following eccentric calf muscle training in chronic Achilles tendinosis. Skeletal Radiol. 2010;39:435-442.

33. Kongsgaard M, Kovanen V, Aagaard P, et al. Corticosteroid injections, eccentric decline squat training and heavy slow resistance training in patellar tendinopathy. Scand J Med Sci Sports. 2009; 19:790-802.

34. Langberg H, Ellingsgaard H, Madsen T, et al. Eccentric rehabilitation exercise increases peritendinous type I collagen synthesis in humans with Achilles tendinosis. Scand J Med Sci Sports. 2007;17:61-66.

35. Mazieres B, Rouanet S, Guillon Y, et al. Topical ketoprofen patch in the treatment of tendinitis: a randomized, double blind, placebo controlled study. J Rheumatol. 2005;32:1563-1570.
36. Petri M, Hufman SL, Waser G, et al. Celecoxib effectively treats patients with acute shoulder tendinitis/bursitis. J Rheumatol. 2004;31:1614-1620.

37. Magra M, Maffulli N. Nonsteroidal antiinflammatory drugs in tendinopathy: friend or foe. Clin J Sport Med. 2006;16:1-3.

38. Heinemeier KM, Øhlenschlæger TF, Mikkelsen UR, et al. Effects of anti-inflammatory (NSAID) treatment on human tendinopathic tissue. J Appl Physiol. 2017;123:1397-1405.

39. Fredriksson M, Li Y, Stalman A, et al. Diclofenac and triamcinolone acetonide impair tenocytic differentiation and promote adipocytic differentiation of mesenchymal stem cells. J Orthop Surg Res. 2013;8:30.

40. Zhang Y, Wang X, Qiu Y, et al. Effect of indomethacin and lactoferrin on human tenocyte proliferation and collagen formation in vitro. Biochem Biophys Res Commun. 2014;454:301-307.

41. Smidt N, van der Windt DA, Assendelft WJ, et al. Corticosteroid injections, physiotherapy, or a wait-and-see policy for lateral epicondylitis: a randomised controlled trial. Lancet. 2002;359:657662.

42. Tonks JH, Pai SK, Murali SR. Steroid injection therapy is the best conservative treatment for lateral epicondylitis: a prospective randomised controlled trial. Int J Clin Pract. 2007;61:240-246.

43. Smidt N, Assendelft WJ, van der Windt DA, et al. Corticosteroid injections for lateral epicondylitis: a systematic review. Pain. 2002;96:23-40.

44. Drug-Induced KK, Disorders T. Adv Exp Med Biol. 2016;920:229-238.

45. Kearney RS, Parsons N, Metcalfe D, et al. Injection therapies for Achilles tendinopathy. Cochrane Database Syst Rev 2015;(5): CD010960.

46. Andres B, Murrell GC. Treatment of tendinopathy: what works, what does not, and what is on the horizon. Clin Orthop Relat Res. 2008;466:1539-1554.

47. Murrell GA, Szabo C, Hannafin JA, et al. Modulation of tendon healing by nitric oxide. Inflamm Res. 1997;46:19-27.

48. Berrazueta JR, Losada A, Poveda J, et al. Successful treatment of shoulder pain syndrome due to supraspinatus tendinitis with transdermal nitroglycerin. A double blind study. Pain. 1996;66:63-67.

49. Paoloni JA, Murrell GA, Burch RM, et al. Randomised, doubleblind, placebo-controlled clinical trial of a new topical glyceryl trinitrate patch for chronic lateral epicondylosis. Br J Sports Med. 2009;43:299-302.

50. Gambito ED, Gonzalez-Suarez CB, Oquinena TI, et al. Evidence on the effectiveness of topical nitroglycerin in the treatment of tendinopathies: a systematic review and meta-analysis. Arch Phys Med Rehabil. 2010;91:1291-1305.

51. Garrick JG. Topical nitroglycerin decreases pain intensity in daily activities: a review. Clin J Sport Med. 2011;21:539-540.

52. Paoloni JA, Murrell GA. Three-year followup study of topical glyceryl trinitrate treatment of chronic noninsertional Achilles tendinopathy. Foot Ankle Int. 2007;28:1064-1068.

53. Distel LM, Best TM. Prolotherapy: a clinical review of its role in treating chronic musculoskeletal pain. PM R. 2011;3(6 Suppl 1): S78-S81.

54. Ryan M, Wong A, Taunton J. Favorable outcomes after sonographically guided intratendinous injection of hyperosmolar dextrose for chronic insertional and midportion achilles tendinosis. Am J Roentgenol. 2010;194:1047-1053. 
55. Scarpone M, Rabago DP, Zgierska A, et al. The efficacy of prolotherapy for lateral epicondylosis: a pilot study. Clin J Sport Med. 2008;18:248-254.

56. Hudgens JL, Sugg KB, Grekin JA, et al. Platelet-rich plasma activates proinflammatory signaling pathways and induces oxidative stress in tendon fibroblasts. Am J Sports Med. 2016;44:19311940.

57. Peerbooms JC, Sluimer J, Bruijn DJ, et al. Positive effect of an autologous platelet concentrate in lateral epicondylitis in a double-blind randomized controlled trial: platelet-rich plasma versus corticosteroid injection with a 1-year follow-up. Am J Sports Med. 2010;38:255-262.

58. Thanasas C, Papadimitriou G, Charalambidis C, et al. Plateletrich plasma versus autologous whole blood for the treatment of chronic lateral elbow epicondylitis: a randomized controlled clinical trial. Am J Sports Med. 2011;39:2130-2134.

59. Tsikopoulos K, Tsikopoulos I, Simeonidis E, et al. The clinical impact of platelet-rich plasma on tendinopathy compared to placebo or dry needling injections: a meta-analysis. Phys Ther Sport. 2016;17:87-94.

60. Maffulli N, Testa V, Capasso G, et al. Results of percutaneous longitudinal tenotomy for Achilles tendinopathy in middle- and long-distance runners. Am J Sports Med. 1997;25:835-840.
61. Maffulli N, Longo UG, Denaro V. Novel approaches for the management of tendinopathy. J Bone Joint Surg Am. 2010;92: 2604-2613.

62. Koh JS, Mohan PC, Howe TS, et al. Fasciotomy and surgical tenotomy for recalcitrant lateral elbow tendinopathy: early clinical experience with a novel device for minimally invasive percutaneous microresection. Am J Sports Med. 2013;41:636-644.

63. Seng C, Mohan PC, Koh SB, et al. Ultrasonic percutaneous tenotomy for recalcitrant lateral elbow tendinopathy: sustainability and sonographic progression at 3 years. Am J Sports Med. 2016;44:504-510.

64. Scott A, Huisman E, Khan K. Conservative treatment of chronic Achilles tendinopathy. CMAJ. 2011;183:1159-1165.

How to cite this article: Mead MP, Gumucio JP, Awan TM, Mendias CL, Sugg KB. Pathogenesis and management of tendinopathies in sports medicine. Transl Sports Med. 2018;1:5-13. https://doi.org/ $10.1002 / \mathrm{tsm} 2.6$ 